\title{
Analysis and Design of Collision Avoidance Optimization Algorithm Based on CSMA/CA
}

\author{
Zhao Gao ${ }^{\text {a }}$, Nan Zhang ${ }^{\mathrm{b}}$ and Hongfeng Han ${ }^{\mathrm{c},}$, \\ International school, Beijing University of Posts and Telecommunications, Beijing 100876, China \\ agaozhao@bupt.edu.cn; b2015212939@bupt.edu.cn;,c,"hanhongfeng@bupt.edu.cn
}

\begin{abstract}
Carrier Sense Multiple Access is a media access control protocol with two contention types in wired network and wireless networks. This paper compares CSMA/CA and CSMA/CD competition modes, introduces and analyzes the existing collision avoidance algorithm, and designs a CSMA/CA collision detection avoidance optimization algorithm based on scheduling algorithm and backoff algorithm. The optimization algorithm focuses on important indicators such as priority, throughput, turnaround time and fair balance and so on, and then the efficiency of the algorithm is further improved.
\end{abstract}

Keywords: Collosion avoidance, wireless network, scheduling algorithm, backoff algorithm.

\section{Introduction}

With the rapid development of Internet technology, more and more devices are connected to the Internet by lots of wired networks and wireless networks. However, although hardware costs are gradually decreasing, conflicts due to shared environments are essential. The CSMA protocol is an improved protocol based on the ALOHA slot. Based on this phenomenon [1], CSMA requires the necessary collision detection mechanism and collision avoidance algorithm to further reduce or avoid the risk of collision [2] [3].

\section{Background}

\subsection{CSMA/CD and CSMA/CA}

\subsubsection{Definition}

CSMA/CD Definition: The bus type network allows only one node (Node) to transmit data at the same time. Once two or more nodes simultaneously transmit data, data collision occurs, and data cannot be normally transmitted and received. The CSMA/CD protocol is to ensure that only one node transmits data at the same time on the network, reducing the probability of data collision.

CSMA/CA Definition: CSMA/CA is a protocol that uses collision avoidance detection to minimize the probability of collision collisions to improve network throughput performance and delay performance.See Table 1 for more application techniques [4].

Table 1. More detailed technologies in CSMA/CA

\begin{tabular}{|c|c|}
\hline Application & WLAN Media Access Control (MAC) layer \\
\hline $\begin{array}{l}\text { Idle evaluation } \\
\text { method }\end{array}$ & $\begin{array}{l}\text { Use the Channel Idle Evaluation (CCA) algorithm to determine if the channel is } \\
\text { idle, by testing the antenna energy and determining the received signal strength } \\
\text { RSSI. }\end{array}$ \\
\hline $\begin{array}{c}\text { Conflict } \\
\text { optimization }\end{array}$ & Reduce conflicts with RTS, CTS, and ACK frames \\
\hline $\begin{array}{l}\text { Encryption } \\
\text { Algorithm }\end{array}$ & Use 64-bit key and RC4 encryption algorithm \\
\hline
\end{tabular}


Table 2. the difference between $\mathrm{CD}$ and CA

\begin{tabular}{|c|c|c|c|c|}
\hline & Defnition & Media & Detection way & $\begin{array}{c}\text { Utilization } \\
\text { rate }\end{array}$ \\
\hline $\begin{array}{c}\text { Collision } \\
\text { deceiton }\end{array}$ & $\begin{array}{c}\text { Carrier sense multi-access with } \\
\text { impact detection, detecting } \\
\text { collisions, avoiding conflicts }\end{array}$ & $\begin{array}{c}\text { Bus } \\
\text { Ethernet }\end{array}$ & $\begin{array}{c}\text { Detected by voltage } \\
\text { changes in the cable } \\
\text { (when the data collides, } \\
\text { the voltage in the cable } \\
\text { changes) }\end{array}$ & $\begin{array}{c}\text { Protocol } \\
\text { channel } \\
\text { utilization is } \\
\text { high }\end{array}$ \\
\hline $\begin{array}{c}\text { Collision } \\
\text { avoidance }\end{array}$ & $\begin{array}{c}\text { Carrier sense multiple access with } \\
\text { collision avoidance, while } \\
\text { transmitting packets can not detect } \\
\text { the presence or absence of conflicts } \\
\text { on the channel, only try to "avoid" }\end{array}$ & $\begin{array}{c}\text { Wireless } \\
\text { LAN }\end{array}$ & $\begin{array}{c}\text { Energy detection (ED), } \\
\text { carrier detection (CS), } \\
\text { energy carrier hybrid } \\
\text { detection - three ways to } \\
\text { detect channel idleness }\end{array}$ & $\begin{array}{c}\text { Low protocol } \\
\text { channel }\end{array}$ \\
\hline utilization \\
\hline
\end{tabular}

\subsubsection{Work Process of CSMA/CD}

The CSMA/CD process has following characters: "Listen firstly, then forward, and forward with listening; Resend after

Delay when collisions are stopped" [5]. See Figure 1 for a schematic of this process.

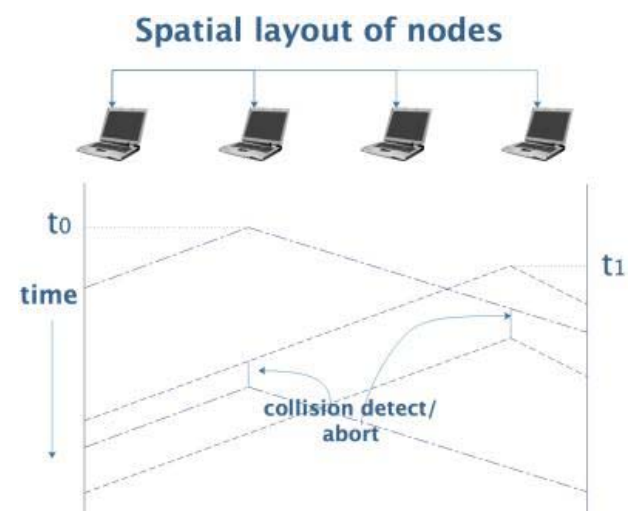

Fig. 1 working process of CSMA/CD

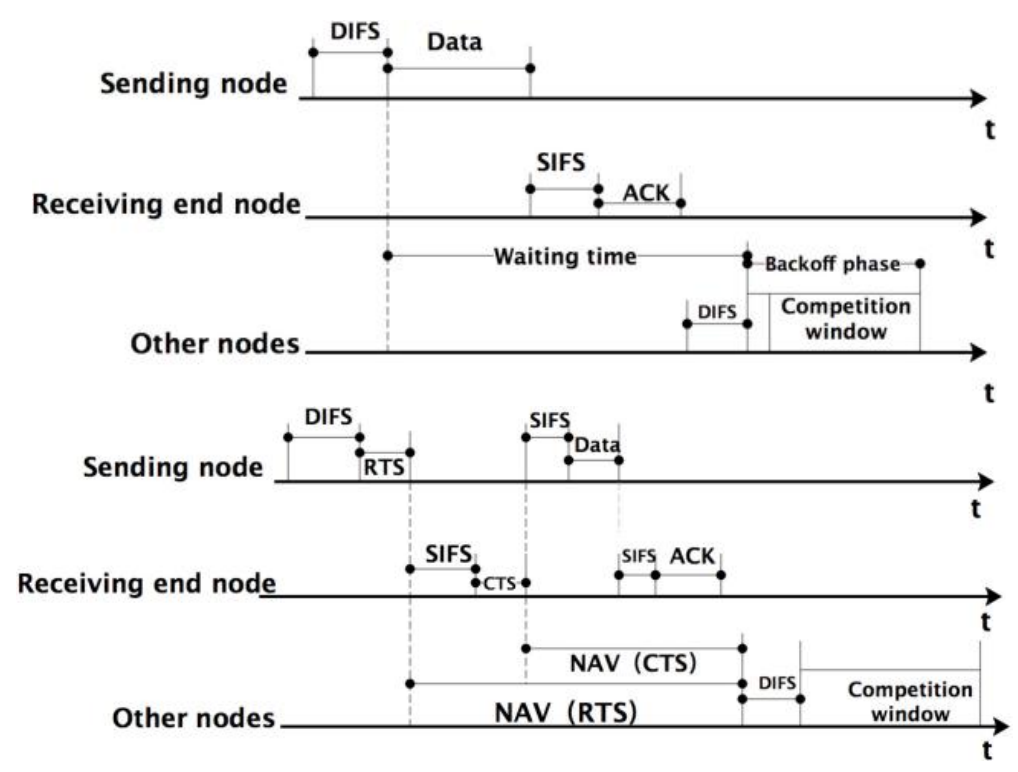

Fig.2 working principle of CSMA/CA 


\subsubsection{Work Process of CSMA/CA}

The source performs channel detection firstly before transmitting data, During this period, it needs to wait for the inter-frame space. For comparison between frames, see Table 3. channel is idle, waiting for DIFS to send data.The receiver sends an ACK to the source via SIFS when received the frame. After the source has experienced DIFS, a contention window appears; If it is detected that the channel is being used, then makes use of the corresponding backoff algorithm. A schematic of this process is shown in Figure 2.

Table 3. Comparison of different inter-frame spacing

SIFS That is, the (shortest) short inter-frame space is used to separate the frames belonging to one
session.

CSMA/CA has two working process [6][7]. In the first process, before transmitting the data, the transmitter detects the channel state. After the channel is idle, waits for a period of time, and then checks whether the channel is idle. If it is still idle, send the data immediately. Otherwise, wait for a certain time randomly. After the time expires, the test is sent again.For the first working process, see Figure 3.In the second process, before the transmitting end sends data, it first detects whether the medium is idle. If the medium is idle, it sends out RTS (Request To Send). After receiving the RTS signal, the receiving end responds to the short signal CTS (Clear To Send). After the CTS packet, the packet is sent. After receiving the data packet, the receiving end will use the value of CRC (Cyclic Redundancy Check) in the data packet to check whether the data is correct. If the test result is correct, the receiving end will respond to the ACK packet and notify the transmission. The end data has been successfully received. When the transmitting end does not receive the ACK packet of the receiving end, the packet is considered to be lost during the transmission, and the packet is retransmitted. Following is the pseudocode. And for the flow chart of this process, see Figure 3.

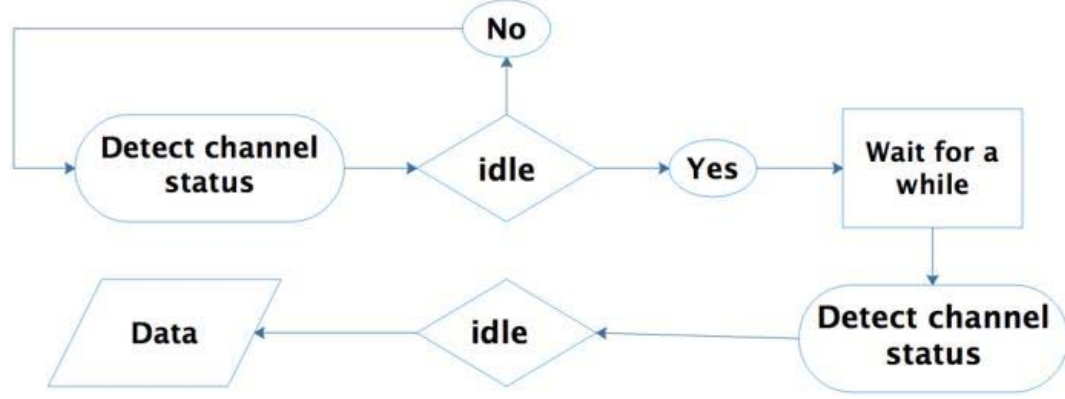

Fig.3 The flowchart of the process

Algorithm:

INPUT:listening

OUTPUT:data(),null

CODE:

boolean flag;

if(flag==true):

back: sent(RTS);

receive(CTS);

sent(data);

float time;

listener(time); 
end;

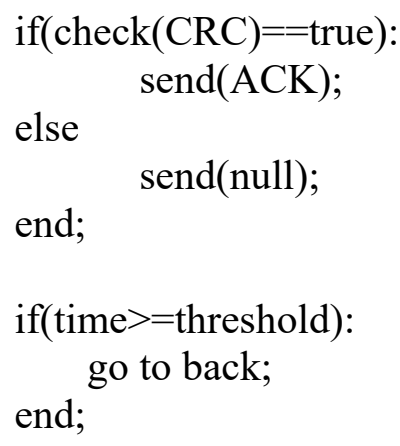

\subsection{Collision}

Collision is a collision between two devices that are trying to transmit data at the same time in the same Ethernet. Collision detection is the detection of the signal voltage on the channel while the computer is transmitting data. When several stations simultaneously transmit data on the bus, the signal voltage swing values on the bus will be linearly superimposed. When the signal voltage swing value detected by a station exceeds a certain threshold, it is considered that at least two stations on the bus are simultaneously transmitting data, that is, a collision occurs. Signals transmitted on the bus are subject to severe distortion due to collisions. The result of the collision is that both frames become useless. When the station detects a collision, it immediately stops transmitting data, and continue to sends broadcast (flooding flood) several bits of human-induced interference signals to other nodes. There are two ways of collision, One way is if there is already data being sent. In the process of transmitting data to $\mathrm{B}, \mathrm{C}$ fails to send data to A. After the failure, $\mathrm{C}$ sends an infection signal to the outside world, so that other signal sources delay sending data to A. In another way, A sends data to $\mathrm{B}$, and $\mathrm{B}$ also sends data to $\mathrm{A}$, and a collision occurs in the channel midway, so that data in both directions fails to be transmitted [8].

\section{Analysis and Design}

\subsection{Truncated Binary Exponential Backoff Algorithm}

Truncated binary exponential backoff algorithm, which is an algorithm used to solve the Ethernet collision problem. When a collision occurs, a random time is postponed, so that the probability of recurrence of collisions during retransmission is reduced.

When the node finds it is busy, it waits for a delay time $\mathrm{M}$ before listening.

The delay time $\mathrm{M}$ is determined by the following algorithm:

A random number between $\mathrm{M}=0 \sim\left(2^{\wedge} \mathrm{k}-1\right)$ multiplied by 512 -bit time (for example, 51.2 microseconds for $10 \mathrm{Mbps}$ Ethernet), $\mathrm{k}$ is the number of collisions (collisions), and the maximum value of $\mathrm{M}$ is 1023 . That is, when $\mathrm{k}=10$ and later, $\mathrm{M}$ is always a product of 51.2 and a random value from 0 to 1023 . When $\mathrm{k}$ is increased to 16 , an error message is issued.

\subsection{Optimization Algorithm Based on Scheduling Algorithm and Backoff Algorithm.}

This paper introduces an optimization algorithm which including scheduling algorithms and backoff algorithm. The first part of the algorithm is the scheduling algorithm, which attempts to solve the problem of collision in a variety of ways. The second part of the algorithm is the backoff algorithm, which attempts to solve the collision problem in the second transmission. The steps of optimization algorithm are:

1. Highest priority scheduling algorithm, which select the signal with the highest priority to execute first.However, it achieving simplicity, but unfairness, can lead to starvation in low-priority. 
2. Then, Shortest Remaining Time Priority. When a newly prepared signal has a shorter completion time than the currently running signal, the system preempts the current signal and selects a new ready signal to execute.

3. Then, Non-Preemptive Shortest Job First. Signal with the shortest completion time takes precedence

4. The last step is non-preemptive first-come-first-served algorithm, which are fair, simple to implement, but short signal behind long signal need to wait for a long time, which is not conducive to user experience.

In some cases, this paper will use the highest corresponding ratio priority algorithm (HRRN), which is a comprehensive algorithm. When scheduling, first calculate the response ratio $\mathrm{R}$ of each process, and then always select the process with the highest $\mathrm{R}$ execution. Response ratio $\mathrm{R}=$ (waiting time + processing time) / processing time

The 4 steps can be seen in the Table 4, and the comparison of features are in the Table 5.

Table 4. Steps algorithms in the optimization algorithm

\begin{tabular}{|c|c|}
\hline Step & Algorithm \\
\hline The first step & Highest priority scheduling algorithm \\
\hline The second step & Shortest Remaining Time Priority. \\
\hline The third step & Non-Preemptive Shortest Job First \\
\hline The fourth step & Non-preemptive first-come-first-served \\
\hline
\end{tabular}

Table 5. Comparison of features of different algorithms

\begin{tabular}{|c|c|c|c|c|c|}
\hline $\begin{array}{c}\text { Scheduling } \\
\text { Algorithm }\end{array}$ & Occupation mode & Throughput & Overhead & $\begin{array}{c}\text { The impact on the } \\
\text { process }\end{array}$ & $\begin{array}{c}\text { Hunger } \\
\text { Problem }\end{array}$ \\
\hline FCFS & Non-preemption & $\begin{array}{c}\text { Not } \\
\text { stressed }\end{array}$ & Minimum & $\begin{array}{c}\text { Unfavorable for } \\
\text { short signals }\end{array}$ & None \\
\hline SJF & Non-preemption & High & $\begin{array}{c}\text { Probably } \\
\text { larger }\end{array}$ & $\begin{array}{c}\text { Unfavorable for } \\
\text { long signals }\end{array}$ & May have \\
\hline SRTN & $\begin{array}{c}\text { Preemption } \\
\text { (Upon arrival) }\end{array}$ & High & $\begin{array}{c}\text { Probably } \\
\text { larger }\end{array}$ & $\begin{array}{c}\text { Unfavorable for } \\
\text { long signals }\end{array}$ & May have \\
\hline HRRN & Non-preemption & High & $\begin{array}{c}\text { Probably } \\
\text { larger }\end{array}$ & Very good balance & None \\
\hline
\end{tabular}

Finally, this paper will extend binary exponential backoff algorithm. However, due to the strict scheduling algorithm in front, the probability of collision in this case is extremely low, so the upper limit 16 in the algorithm is modified to 10 , that is, when the number of collisions exceeds 10 times, the transmission fails, the transmitted frame is discarded, and the transmission error is sent.

\section{Summary}

This paper focuses on the analysis and design of collision avoidance optimization algorithm based on CSMA/CA. This paper compares CSMA/CA and CSMA/CD within work process, and analysis the influence of collision. The traditional truncated binary exponential backoff algorithm has its advantages and disadvantages. Based on this, a new optimization algorithm is designed based on the 
scheduling algorithm and the backoff algorithm. The performance of the algorithm is further improved by considering various important performance indicators.

\section{References}

[1]. Laufer R, Kleinrock L. The Capacity of Wireless CSMA/CA Networks [J]. IEEE/ACM Transactions on Networking, 2016, 24(3):1518-1532.

[2]. Bianchi G, Fratta L, Oliveri M. Performance evaluation and enhancement of the CSMA/CA MAC protocol for 802.11 wireless LANs[C]// IEEE International Symposium on Personal, Indoor and Mobile Radio Communications. IEEE, 2002:392-396 vol.2.

[3]. Soh K H, Chia H P, Dakhane K, et al. CSMA/CD wireless LAN: US, US 6539028 B1[P]. 2003.

[4]. Ming-Ming L I, Chen G. An Improved FQ-CSMA Protocol for Real-Time Monitoring in WSN[J]. Computer Development \& Applications, 2012.

[5]. Ieee B E. Amendment to Carrier Sense Multiple Access With Collision Detection (CSMA/CD) Access Method and Physical Layer Specifications-Aggregation of Multiple Link Segments[J]. IEEE Std 802.3ad-2000, 2000:i-173.

[6]. Park T R, Kim T H, Choi J Y, et al. Throughput and energy consumption analysis of IEEE 802.15.4 slotted CSMA/CA[J]. 2005, 41(18):1017-1019.

[7]. Sabharwal A, Khoshnevis A, Knightly E. Opportunistic spectral usage: bounds and a multi-band CSMA/CA protocol[J]. Networking IEEE/ACM Transactions on, 2006, 15(3):533-545.

[8]. Nilsson T, Wikstrand G, Eriksson J. A collision detection method for multicast transmissions in CSMA/CA networks: Research Articles[M]. John Wiley and Sons Ltd. 2007. 